González, J. (2015). Innovación y tecnología, factores claves de competitividad empresarial. Una mirada desde lo local. Revista Lebret, 7. Bucaramanga, Colombia: Universidad Santo Tomás, pp. 103 - 124. ISSN: 2145-5996.

\title{
Innovación y tecnología, factores claves de competitividad empresarial. Una mirada desde lo local*
}

\section{Innovation and technology, business competitiveness key factors. A look from the local level.}

Julio Alfonso González Mendoza ${ }^{I}$

\begin{abstract}
Resumen
Este trabajo de revisión y discusión, tiene como objetivo realizar un análisis de los niveles de innovación en Colombia y Norte de Santander. La metodología es documental y los principales resultados señalan que el nivel de innovación de Colombia, está por debajo del promedio de América Latina y muy lejos de Estados Unidos y Canadá. Norte de Santander muestra indicadores por debajo del promedio nacional y sus principales problemas son la falta de capital humano, escasos recursos financieros, deficiente información de financiación pública y beneficios tributarios, además de la desarticulación entre los actores del sistema.
\end{abstract}

\section{Palabras clave}

Innovación y tecnología, competitividad, nivel de innovación, medición de innovación.

\section{Códigos de clasificación JEL: O3, O32, O38, L8}

\begin{abstract}
This review and discussion work, aims to make an analysis of the levels of innovation in Colombia and Norte de Santander. The methodology is documentary and the main results indicate that the level of innovation in Colombia is below the average for Latin America and far from the United States and Canada. Norte de Santander shows indicators below the national average and its main problems are the lack of human capital, limited financial resources, poor information of public funding and tax benefits, and the lack of coordination between the actors of the system.
\end{abstract}

\section{Keywords}

Innovation and technology, competitiveness, level of innovation, innovation measurement

\footnotetext{
* Este artículo deriva de la investigación "Innovación y tecnología de las empresas manufactureras de Norte de Santander. Una mirada desde lo local", financiada por la Universidad Francisco de Paula Santander.

1 Magíster en Planeación. Docente Universidad Francisco de Paula Santander, Cúcuta-Colombia. Correo electrónico: alfonsogonzalez@ufps.edu.co
} 


\section{Introducción}

La innovación tecnológica ha sido un tema recurrente cuando se trata de identificar los factores de competitividad que inciden en el impulso a las actividades generadoras de valor, el crecimiento económico y el desarrollo de una nación (Departamento Administrativo Nacional de Estadística, 2006, p. 213).

Los Estados, los sectores económicos y las empresas cada vez deben ser más competitivos, y eso implica que sus aparatos productivos y su cultura empresarial estén en continua adaptación a un mundo cambiante y turbulento, en búsqueda de nuevas prácticas que den ventaja competitiva y logren entregar un producto o servicio con la misma calidad que sus competidores, pero con menores costos de producción.

La búsqueda de una identidad innovadora y competitiva requiere del compromiso de los actores del sistema: el Estado generando políticas de ciencia y tecnología que favorezcan la cultura empresarial; las empresas generando estrategias necesarias para ser eficientes y afrontar la competencia global, las universidades aportando su experiencia en investigación y desarrollo, y los sectores económicos propiciando el establecimiento de redes de colaboración y transferencia de conocimientos. Cada actor desempeña un rol en esta dinámica y sus esfuerzos no pueden ser individuales, sino que debe haber una adecuada cohesión y armonía entre ellos, para sumar esfuerzos y alcanzar objetivos.

No obstante, algunos indicadores muestran que la tarea no se está cumpliendo y pese a que se han establecido mecanismos para incentivar la innovación y la tecnología empresarial, siguen las empresas colombianas rezagadas con respecto a sus similares, en países de la región y muy lejos de los desarrollados, por lo que convendría indagar sobre la existencia, efectividad, coherencia y condición integradora de los mecanismos establecidos.

Colombia es un país con vocación de libre mercado; actualmente tiene 16 tratados comerciales con diferentes países de América, Europa y Asia (Ministerio de Industria y Comercio, 2014), por lo que su aparato productivo debe ser altamente competitivo para enfrentar las multinacionales en su propio territorio y además para intentar llegar con éxito a los mercados mundiales.

Este trabajo consiste en una revisión documental con el objetivo de analizar los niveles de innovación en Colombia y del departamento Norte de Santander, explicando conceptos de innovación y tecnología, la imbricación existente entre ellos y su influencia en la competitividad empresarial.

Se presenta un breve panorama sobre la innovación y la tecnología a nivel mundial, luego se contextualiza a nivel de Colombia para finalmente explicar el caso de Norte de Santander. En la actualidad, se desarrollan investigaciones sobre los niveles de tecnología e innovación en cada uno de los sectores de la economía departamental, 
teniendo especial cuidado de presentar resultados que permitan comparabilidad y trazabilidad con otras mediciones nacionales y globales.

Igualmente se recopila y analiza la normativa colombiana y nortesantanderana sobre las intenciones de hacer que investigación, tecnología e innovación generen competitividad, desarrollo y bienestar a la sociedad nacional y regional. Finalmente, se desarrolla el tema de medición de innovación y tecnología, desde las propuestas de Pavitt, Manuales de Frascati, Oslo y Bogotá mostrando algunas mediciones realizadas en Colombia a través del DANE y otras correspondientes al sector cerámico en Norte de Santander.

Para el desarrollo de este artículo de revisión se utilizaron fuentes documentales. Se realizó una búsqueda sistemática en las bases de datos Science Direct, Proquest, Scopus, Scielo y Redalyc, con las palabras innovación y tecnología, competitividad, medición de innovación y tecnología, encontrando cerca de doscientos documentos, posteriormente se clasificaron por tema, quedando cerca de cincuenta, los cuales se revisaron y analizaron, para la construcción de este documento.

Una búsqueda en fuentes secundarias se llevó a cabo para obtener normativa y estadísticas de Colombia y de Norte de Santander, algunos datos se solicitaron directamente al Departamento Nacional de Estadísticas (DANE), Departamento Nacional de Planeación (DNP) y Cámaras de Comercio.

\section{Innovación y tecnología empresarial}

\section{Teorías de innovación: producto comercial o conocimiento}

Distintos autores han escrito teorías de innovación como componente fundamental de la competitividad empresarial; algunas afirman que el fin de toda innovación es un producto comercial que impacte positivamente en lo económico y de posición estratégica a la empresa, mientras que otras argumentan que no necesariamente el fin es el beneficio económico, sino la generación de nuevas teorías y a través de ellas, llegar inclusive al descubrimiento de nuevas disciplinas de la ciencias (Kline \& Rosenberg, 1986).

Las teorías de innovación pueden clasificarse en lineales y de sistemas. Las lineales orientan los procesos de innovación hacia la oferta mientras que las de sistemas tienen una orientación hacia la demanda (Leif, 1999).

La visión lineal de innovación explica que la ciencia produce tecnología y esta satisface necesidades del cliente (Kline \& Rosenberg, 1986), tienen el propósito de generar aplicaciones comerciales y se utilizan únicamente con el fin de crear o mejorar productos; así la investigación y por tanto la ciencia siempre conduce a un producto comercial y solo se convierte en innovación cuando genera retorno y utilidades a la empresa. Según Rothwell (1994) y Forrest (1991), esta visión es considerada de 
primera y segunda generación y es descrita como un proceso de conversión, en el que unas entradas se convierten en productos, a lo largo de un proceso elaborador.

Por su parte, la visión de innovación como sistemas, reconoce explícitamente la compleja relación entre los elementos del proceso innovador, tales como: fuentes, procesos, elementos elaboradores, productos, recursos y actores que intervienen es su generación y aplicación. Esta interacción entre los distintos actores y articulación de recursos y procesos es conocida como redes de innovación (Freeman, 1991). Las principales características del enfoque de sistemas son:

a) Coloca la innovación y el aprendizaje como centro del proceso; b) se considera holística e interdisciplinaria, ya que incluye una gran cantidad de determinantes de la innovación, no solo los factores económicos, sino los organizacionales, sociales, políticos, económicos, entre otros; c) emplea perspectivas históricas, debido a que los procesos de innovación se desarrollan con el tiempo, y conforme a las condiciones sociales del momento; d) hace énfasis en las diferencias entre los sistemas, comparando los existentes entre sí, en lugar de hacerlo entre los reales e ideales; e) énfasis en la interdependencia y la no linealidad. Considera que las empresas no hacen un proceso de innovación de manera independiente, sino que interactúan con otras organizaciones de igual condición o del entorno como el caso del Estado (Edquist \& Johnson, 1997); y f) están asociados con diversidad conceptual, por lo tanto, un mayor desarrollo innovador, implica avanzar desde el pluralismo, hasta una especificación clara y puntual del proceso en cuestión.

Existen varios subsistemas dentro de la teoría de sistemas de innovación: encadenamiento, proceso distribuido, aprendizaje interactivo y de red. El modelo de encadenamiento hace énfasis en que la gestión de la innovación, incluida la política pública, debe reconocer las fortalezas de las empresas del sector y tratar de coordinar los esfuerzos a través de cadenas de innovación, conformadas por varias empresas con intereses similares (Leif, 1999).

Modelo distribuido: este modelo contradice la creencia que se tuvo durante años que las innovaciones se desarrollan por los fabricantes de productos, y propone un proceso distribuido, en que las innovaciones pueden proceder de al menos tres fuentes distintas o de sus combinaciones: proveedores, productores y usuarios (Von Hippel, 1988).

La teoría del aprendizaje interactivo: parte de la crítica a las ideas ortodoxas, en el que innovar se hace mediante el proceso de aprender haciendo (Arrow, 1962). La idea central de la innovación no depende del productor, sino que se hace con base en información de necesidades del cliente y una manera eficiente de hacerlo es a través de asociaciones de clientes o grupos de interés reconocidos sobre el producto. El establecimiento de una adecuada relación entre productor y usuario implica una buena comunicación y discusión sobre las necesidades y deficiencias de un producto y la búsqueda de soluciones que la superen. 
El Modelo en Red: subraya que el aprendizaje nace en las empresas y que la innovación es fundamentalmente un proceso colaborativo en red (Hobday, 2005), apoyada en herramientas informáticas que vuelven a la empresas más veloz y eficiente en el desarrollo de nuevos productos o mejora a los existentes (Rothwell, 1994).

El proceso innovador, según la teoría de sistemas tiene múltiples variables presentes en el entorno y la empresa, no solamente depende de su vocación innovadora y capacidades internas, sino de las condiciones que tenga el sector, de las políticas gubernamentales y de las alianzas que la empresa pueda establecer con el Estado y con instituciones de reconocido prestigio en investigación, como las universidades. La capacidad de establecer relaciones, interdependencias, consolidar alianzas y aprovechar las políticas y normas a su favor, dará una adecuada condición para que el proceso de innovación sea exitoso.

Otros estudios han tratado de ir más allá de la perspectiva basada en la fabricación de productos y tratan de abordar las particularidades del servicio al cliente, tal es el caso de Gallouj y Weinstein (1997), quienes proponen tres enfoques de innovación: asimilación, demarcación e integración.

El enfoque de asimilación se percibe de forma similar a la innovación manufacturera y reduce la innovación a la adopción y uso de la tecnología. El ciclo de vida del producto comienza con la introducción de innovaciones en los procesos que tienen como objetivo mejorar la eficiencia continua, para optimizar la calidad de los servicios e incrementar nuevos productos.

El enfoque de demarcación se centra en la producción no tecnológica, es decir, basada en servicios o innovación invisible, por ejemplo, la personalización del servicio, resolución de problemas, nuevos métodos y nuevas estructuras organizativas. Estas innovaciones no están presentes en el producto, pero el cliente las recibe en forma de servicio a la hora de la venta o posventa.

El enfoque de integración abarca tanto los bienes y servicios como los modos tecnológicos y no tecnológicos. En este nuevo contexto dos cambios principales se llevan a cabo: la fabricación actúa cada vez más sobre los servicios y los servicios son cada vez más influyentes en la fabricación.

Por ser la teoría de sistemas una compleja relación entre actores que requieren una articulación y cohesión de esfuerzos, ya que no se puede realizar el proceso de manera independiente, las discusiones posteriores, en este trabajo, se harán bajo esta visión, igualmente será necesario apoyarse el enfoque integrador, ya que los servicios que se ofrecen al cliente son definitivos para la competitividad empresarial.

\section{Innovación y tecnología, factores claves de competitividad empresarial}

En el mundo moderno, la competitividad es el principal desafío para triunfar en los negocios, ser competitivo es tener la capacidad de proveer un producto o 
servicio comparable al de sus rivales, pero realizando actividades de una manera más eficiente o desarrollando métodos únicos y exclusivos de la empresa que permitan crear un mayor valor agregado y el cliente lo pueda percibir en términos de precio, oportunidad y calidad.

Respecto de la competitividad, Boza y Pérez (1996) proponen tres explicaciones vistas desde el ámbito en que se realiza: a) empresa, b) sector industrial y c) a nivel macro de la economía. En el ámbito de la empresa consiste ser capaz de suministrar bienes y servicios de la misma calidad o mejor que sus competidores (Enright, Frances \& Scott, 1994). Dentro de la industria, la competitividad significa producir bienes con los estándares de calidad requeridos en el mercado, pero con costos iguales o inferiores a los de industrias similares del resto del mundo, comparados en un determinado período de tiempo (Haguenauer, 1989). Desde el punto de vista nacional, la economía es competitiva si es capaz de incrementar o sostener la participación en los mercados internacionales y además producir un mejoramiento del nivel de vida de sus habitantes (Porter, 1990).

¿Y cómo una empresa, sector económico o nación pueden ser competitivos?

La competitividad puede provenir de diversas fuentes, entre las que destacan, la innovación y la tecnología, constituyéndose junto al capital humano en determinantes de la productividad, de la estructura de costos de la empresa, de los precios de los productos y de los salarios de los trabajadores, todo lo cual repercute en los niveles de empleo, en el crecimiento económico nacional y en la calidad de vida de la población (Peñaloza, 2007, p. 81).

Listerri \& Angelelli (2002) afirman que para incrementar la competitividad las empresas deben establecer estrategias, tales como: a) Mejorar el entorno en el que se desempeñan; b) Consolidar los mercados que han conquistado; c) Desarrollar nuevos mercados de productos o servicios; d) Fortalecer cadenas productivas y redes de apoyo empresarial; e) Fomentar la empresarialidad y, f) Fomentar la innovación y desarrollo tecnológicos.

Como se observa son diferentes las acciones o estrategias para ser competitivo, pero como lo explican diferentes autores la competitividad empresarial encuentra en la innovación y la tecnología sus principales aliados, por ello se discutirán acerca de sus características, interacción con otros actores, niveles, fuentes, clases y formas de medición en las siguientes secciones.

Según la Organización para la Cooperación y el Desarrollo Económico (OCDE, 2005), la innovación es la creación o mejoramiento de productos, procesos, métodos de comercialización o métodos organizativos (gestión) en las prácticas internas de la empresa, con el fin de generar competitividad en el mercado (Comisión Regional de Competitividad Norte de Santander, 2007).

Nagles (2007) afirma que la innovación es la "capacidad de la empresa para generar soluciones ingeniosas, creativas y rentables de manera que atienda las necesidades, 
expectativas y demandas de los consumidores, mercados y sociedad en general" ( $\mathrm{p}$. 82). Por su parte, Ochoa (2007) define innovación como el proceso sistémico, "que requiere de asociaciones y vínculos entre las distintas áreas del conocimiento, para la implementación y el funcionamiento de mejoras en las formas de hacer las cosas, sobre la base de ideas creativas y transformadoras" (p. 2), con el fin de llegar con éxito a los mercados y lograr en ellos un impacto social, económico o ambiental.

Para Schumpeter (1978), la innovación es la fuerza principal que impulsa el sistema capitalista y que permite su transformación y desarrollo económico y social. Son amplias y diversas las definiciones de innovación, pero todas confluyen en las estrategias y acciones que la empresa desarrolla para dar soluciones creativas al cliente, a partir de sus requerimientos o con el objetivo de generar necesidades de consumo antes inexistentes.

En consecuencia, las estrategias y acciones que se implementen deben estar enmarcadas en un conocimiento adecuado con el entorno en que se desempeñan las compañías, aprovechando las políticas del Estado y estableciendo alianzas estratégicas o redes de colaboración con otros actores, para potenciar sus fortalezas y minimizar sus debilidades. Esas estrategias y acciones son generadas desde cuatro perspectivas, que a su vez se convierten en el origen de la innovación: el producto, los procesos, el mercado y las decisiones organizacionales (gestión).

\section{Niveles, fuentes y clases de innovación empresarial}

Con el fin de contextualizar acerca de los niveles, fuentes y clases de innovación en las empresas, se explica brevemente cada una de estas taxonomías, abordando la temática desde los autores más representativos. Así, Pineda (2009) plantea tres niveles que deben tenerse en cuenta al momento de afrontar un proceso de innovación: 1) el nivel macroeconómico se refiere a las políticas de ciencia y tecnología que desarrollan los Estados para propiciar condiciones favorables a la innovación; 2) el nivel mesoeconómico consiste en la estructura del sistema, la articulación de sus actores y cohesión con el entorno para desarrollar las políticas y propuestas en nivel macroeconómico; y 3) el nivel microeconómico hace referencia a las acciones que las empresas ejecutan y que se convierten en el principal elemento dinamizador del proceso de innovación.

De esta forma, el proceso innovador de las empresas está influido por las condiciones de la sociedad, tales como los factores sociales, políticos, legales y económicos, por ende, la innovación es favorecida o entorpecida por las decisiones de los gobiernos, las políticas de los gremios y las estrategias de las empresas en particular.

En el interior de la compañía, "el nivel de capacidad de innovación y tecnología de una empresa o sector, debe ser el punto de partida para la definición de la estrategia competitiva, ya que esto permite conocer las fortalezas y debilidades que condicionan 
su desempeño" (Arzola, 2007, p. 4); esto constituye un aspecto necesario y relevante en el diagnóstico y establecimiento de un plan estratégico empresarial, que a su vez conduce al descubrimiento de las fuentes y motivaciones iniciales de innovación.

Respecto de las fuentes de innovación, Drucker (2002) plantea que existen unas internas y otras externas a la compañía. Las internas son: a) el éxito o fracaso inesperado causado por algún hecho del entorno externo; b) las diferencias observadas entre las necesidades del cliente y las características de los productos o servicios ofrecidos; c) la necesidad de nuevos procesos internos o la redefinición de los mismos, por la deficiencia de alguna de sus partes; y d) los cambios del mercado causados por desgaste del ciclo del producto y el cambio de hábito de los clientes.

Las fuentes externas, continúa Drucker, son las originadas por: e) los cambios de valores y percepciones de la sociedad; f) las variaciones demográficas que afectarán a la sociedad, la economía y la empresa y g) las nuevas innovaciones y tecnologías que afectan a la mayoría de los procesos de las empresas (tabla 1).

Tabla 1. Fuentes de innovación

\begin{tabular}{ll}
\hline \multicolumn{1}{c}{ INTERNA } & \multicolumn{1}{c}{ EXTERNA } \\
\hline $\begin{array}{l}\text { La sorpresa: Éxito o fracaso por algún hecho } \\
\text { inesperado del medio }\end{array}$ & $\begin{array}{l}\text { Los cambios en el mercado como consecuencia del } \\
\text { ciclo de vida del producto }\end{array}$ \\
$\begin{array}{l}\text { Disonancia entre la realidad y el cliente } \\
\text { Cambios en la sociedad } \\
\text { Necesidad de procesos internos en la empresa y } \\
\text { eslabones débiles }\end{array}$ & Variaciones demográficas \\
\hline
\end{tabular}

Fuente: El autor a partir de Drucker, 2002.

Cada uno de estos factores puede estar interrelacionado o superpuesto con otros y presentarse de forma simultánea que motiven la investigación, el desarrollo y el tránsito hacia la generación de un nuevo conocimiento.

Para López et at. (2004), las fuentes principales de innovación se encuentran en el interior de la industria en: a) actividades de investigación y desarrollo, b) departamento de producción, c) trabajadores, d) errores y deficiencias de los producto/servicios. $\mathrm{Y}$ en el entorno, es decir, como factores externos en e) los clientes. f) compra de tecnología como son los contratos de licencia, servicios técnicos, adquisición de patentes y, g) información proveniente de otras organizaciones empresariales, institutos de investigación, universidades y de la competencia.

Tomando los conceptos de Drucker y López y relacionándolos con la teoría de sistemas, la innovación proviene de diversas fuentes: internas y externas, la interacción con el medio y un proceso elaborador que genera nuevos conocimientos representados en procesos, productos, marketing o gestión (figura 1). 
Figura 1. Fuentes de innovación

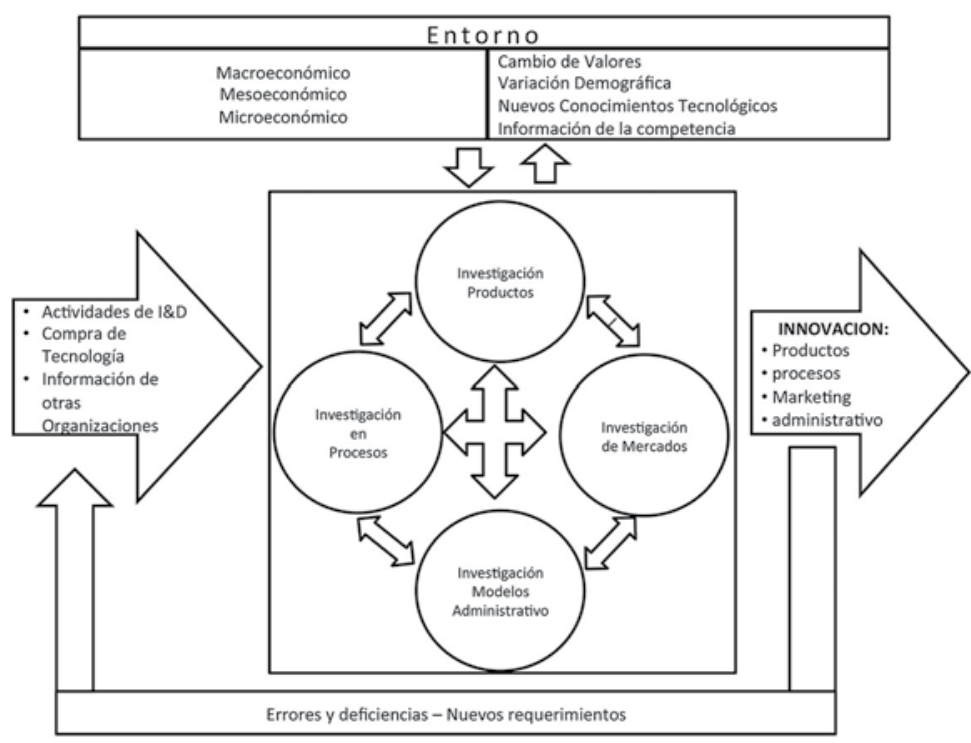

Fuente: El autor.

Cuando se habla de la clase de innovación se refiere a pequeños o grandes cambios en la funcionalidad de un proceso, producto o práctica administrativa o de mercados. Existen varias taxonomías, una de las cuales consiste en valorar la magnitud del cambio o impacto que conlleven, así: puede presentarse una transformación total y absoluta de las actividades realizadas en la empresa o presentación de un producto novedoso, o más sencillas que impliquen solo cambios leves en el actuar empresarial o en el producto final. Bajo este concepto Dussauge, Hart \& Ramantsoa (1992) presentan la siguiente clasificación de innovación: a) incremental donde la relación entre componentes y arquitectura del producto no se cambia, solo se mejoran levemente; b) radical, en la que la arquitectura y los componentes del producto son modificados sustancialmente dando origen a un nuevo producto; b) modular, donde se cambian radicalmente los componentes del producto, pero su arquitectura permanece; c) arquitectural, en la que se modifica la forma en que se relacionan los componentes y conceptos del producto, pero estos permanecen sin cambio.

Otra forma de clasificar la innovación es tomando en cuenta su naturaleza, y para ello, Damanpour y Gopalakrishnan (1998) la clasifican como tecnológica y no tecnológica (administrativa). La innovación tecnológica consiste en los cambios realizados a los productos y a los procesos. La no tecnológica comprende las innovaciones que no se incluyen en los productos, y principalmente se da como 
mejoras en las prácticas comerciales, financieras y organizacionales que potencian la competitividad de la empresa (figura 2).

Figura 2. Innovación y tecnología

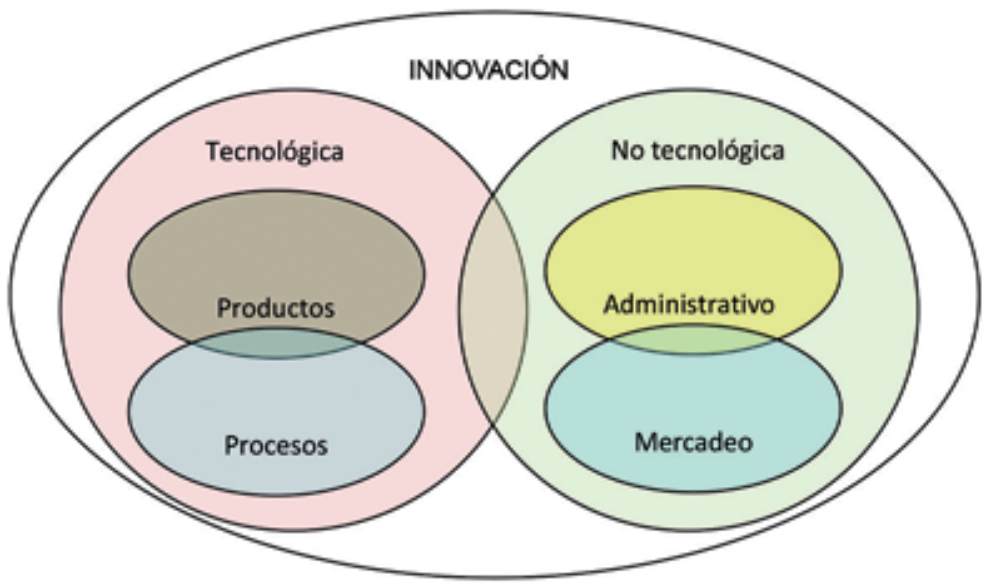

Fuente: El autor.

Como se ha dicho, la innovación tecnología es una forma de lograr innovación, por lo que es conveniente contextualizar su dimensión y la imbricación existente entre los dos conceptos. Es muy frecuente utilizar el término innovación, tecnología o innovación tecnológica para hacer referencia al mismo concepto, y en ocasiones pareciera ser más una discusión de tipo semántico. Innovar consiste en generar conocimiento, y algunas veces, nuevas tecnologías que pueden ser aplicadas a productos, procesos de producción y gestión. "La tecnología se define como un conjunto de técnicas industriales susceptibles de ser aplicadas a un proceso productivo. La innovación puede implicar cambios profundos basados en avances científicos y tecnológicos. En este caso se habla de innovación tecnológica” (López et at., 2004, p. 10).

Para Peñaloza (2007), la tecnología se entiende como los conocimientos organizados y orientados a la resolución de un problema concreto; es la aplicación eficaz y eficiente de una serie de técnicas, conocimientos y experiencias a situaciones que requieren ingenio para su solución mientras que (Benavides, 1998) cree que tecnología es el sistema de conocimientos y de información derivado de la investigación, de la experimentación o de la experiencia y que, unido a los métodos de producción, comercialización y gestión, permite crear nuevos o mejorados productos, procesos o servicios.

Las tecnologías que aportan innovación a la empresa, pueden clasificarse, a su vez, como blandas y duras. Según Guevara (2000) las tecnologías blandas son 
los conocimientos aplicados al direccionamiento de la organización, a los métodos aplicados en la compañía para realizar operaciones y para administrar los recursos con el fin de hacer un producto o servicio que satisfaga las necesidades del cliente. $\mathrm{Y}$ las tecnologías duras son las representadas en equipos, maquinarias y procesos productivos.

Una de las tecnologías blandas es la información, que Castells (1999) define como un conjunto convergente de tecnologías de la microelectrónica, la informática, las telecomunicaciones, la televisión, la radio y la optoelectrónica, y que ocupan una franja importante de la revolución tecnológica moderna.

\section{Breve panorama de innovación y tecnología en Colombia}

La quinta edición de The Global Innovation Index 2012 (Stronger Innovation Linkages for Global Growth), presentada por la Organización Mundial de la Propiedad Intelectual de las Naciones Unidas (OMPI) y la escuela de negocios INSEAD, publicó el ranking de 141 países respecto de su capacidad de innovación. Lo preocupante de este informe es que los países del área latinoamericana no aparecen entre los mejores clasificados y casi todos están en la segunda parte de la tabla. El ranking de los países de esta región lo encabeza Chile en el puesto 39; Brasil 58; Costa Rica 60; Colombia 65; Uruguay 67; Argentina 70, Perú 75 y Venezuela 118.

Igualmente, el informe de Red de Indicadores de Ciencia y Tecnología Iberoamericana e Interamericana (RICYT) de 2012 da a conocer el porcentaje del PIB que los países invierten en ciencia y tecnología: en el 2010 Brasil invirtió $1.16 \%$; Argentina $0.62 \%$; Cuba $0.61 \%$; México $0.48 \%$, y Colombia $0.19 \%$; el promedio de América Latina y el Caribe (ALC) es de $0.75 \%$, mientras que, Estados Unidos invierte $2.89 \%$ y Canadá $1.8 \%$.

El panorama para Colombia es muy desalentador, ya que está por debajo del promedio de ALC y muy lejos de Estados Unidos y Canadá, con el agravante que no es una situación fortuita o de política del gobierno de turno, sino que es tendencia de los últimos 15 años. Además, prácticamente toda la investigación se origina en las universidades y centros de investigación y poco por la empresa privada. El sector privado "aún no cree en la investigación y mucho menos en hacer innovación y se limita a realizar actividades de supervivencia comercial y manufacturera" (Argel, 2009, p. 255).

El Departamento Nacional de Estadísticas (DANE) mide la innovación en Colombia cada dos años mediante la Encuesta de Innovación y Tecnología - EDIT, se extraen algunos datos de 2013 que permite entender las dificultades del sistema.

Según la EDIT IV (DANE, 2013) en el periodo 2011-2013 se realizaron en Colombia 1808 innovaciones, repartidas así: 44\% organizacionales, $42 \%$ productos o servicios mejorados, $10 \%$ proceso de comercialización y $4 \%$ nuevos productos o 
servicios; comparada con la encuesta anterior EDIT III (DANE, 2011) se encuentra que hubo una reducción del $63 \%$ del total de las innovaciones. El informe muestra que 236 empresas obtuvieron registros de calidad, de las cuales el 79\% lo hicieron en procesos y $21 \%$ en productos. La mayor proporción de innovación se realizó en aspectos organizaciones, impulsadas por las certificaciones de calidad obtenidas en este periodo, mientras que las innovaciones de productos nuevos solo ocupan una pequeña porción.

El personal que las empresas utilizan para la realización de actividades de innovación tienen formación académica, así: profesional $28 \%$, especialización $15 \%$, magíster $14 \%$ y doctorado $7 \%$, el resto de personal posee formación de tecnología, tecnólogo o egresados SENA. Uno de los problemas que presentan las empresas para realizar investigación es la falta de personal con competencias en investigación, es decir, doctorado y maestría, lo cual no permite que la innovación tenga mayor cantidad y calidad.

Las principales fuentes de innovación son: $37 \%$ de los directivos, $18 \%$ del departamento de ventas, $16 \%$ de otro departamento, $11 \%$ del departamento de producción, $7 \%$ del departamento de $\mathrm{I}+\mathrm{D}, 6 \%$ de grupos interdisciplinarios y $2 \%$ de casa matriz. Estos resultados señalan que la mayor fuente de innovación surgen a partir de ideas e iniciativas gerenciales, mientras que los departamentos de I+D no aportan significativamente al proceso.

Respecto a la inversión que hacen las empresas, la EDIT IV señala que las fuentes de financiación fueron: $74 \%$ recursos propios, $11 \%$ recursos públicos, $8 \%$ banca privada, $5 \%$ cooperación y donaciones, las demás inversiones provienen de otras empresas, de fondos privados y de otras empresas del mismo grupo empresarial. Las compañías saben que la innovación es una de las principales fuentes de competitividad, y por ello, el esfuerzo que hace de innovar con sus propios recursos es justificable, lo que no parece coherente es que la inversión de recursos públicos sea tan baja, principalmente debido al excesivo trámite y a las dificultades para cumplir con todos los requisitos exigidos.

Los principales obstáculos encontrados para realizar innovación son: falta de recursos, falta de personal calificado, incertidumbre frente a la demanda de los servicios o productos innovados, incertidumbre frente a la ejecución de una técnica innovada y dificultad en el cumplimiento de las regulaciones.

Para acceder a los recursos públicos, las empresas encontraron otras barreras, como: tiempo del trámite excesivo $20 \%$, dificultad para cumplir con los requisitos o completar trámites $18 \%$, condiciones de financiación poco atractivas $16 \%$, demora en la intermediación entre banca comercial y líneas públicas de crédito $16 \%$, desconocimiento de las líneas de financiación pública existentes $15 \%$ y falta de información sobre requisitos y trámites $15 \%$. Adicional a estas dificultades, el $56 \%$ 
de las empresas que innovaron y posteriormente solicitaron beneficios tributarios tuvieron algún obstáculo relacionado con falta de información, plataforma en línea, dificultad de diligenciamiento, trámites excesivos, tiempo excesivo en el trámite y poco beneficio, lo cual desmotiva al empresario y hace que el proceso innovador en Colombia no tenga mayor dinamismo.

Como se observa, la empresa privada y el Estado hacen esfuerzos para que el sistema funcione efectivamente, sin embargo, las encuestas muestran que los resultados no son los deseados y que hay enormes dificultades para hacer que los mecanismos creados se articulen adecuadamente y produzcan la sinergia necesaria para que el sector productivo sea innovador, competitivo y capaz de enfrentar los mercados globales.

\section{La innovación de la empresa nortesantandereana}

El Departamento Administrativo de Ciencia y Tecnología - Colciencias (2010) señala que para el 2010, el departamento de Norte de Santander tuvo una participación de $0.81 \%$ del total nacional en actividades de ciencia, tecnología e innovación (ACTI), mostrando un leve crecimiento en los últimos diez años. El valor ejecutado fue de 17397 millones de pesos y las instituciones ejecutoras fueron: empresas, $31.2 \%$; instituciones de educación superior, 54.6\%; entidades gubernamentales, $8.6 \%$; centros de investigación y desarrollo tecnológico, $1.6 \%$.

Una de las formas de medir la innovación es a partir del porcentaje de recursos destinados a ella y por los productos y procesos que se han obtenido de este ejercicio, por tanto, considerando la información referida, se puede inferir que el nivel de innovación e investigación del departamento Norte de Santander está por debajo del promedio nacional, inclusive más abajo del puesto que ocupa por aporte al PIB nacional que es del 1,7\% (Departamento Norte de Santander, 2012).

Apenas el $0.5 \%$ de los proyectos aprobados por Colciencias son de Norte de Santander, mientras que el promedio nacional es de $2,5 \%$, cifras que reflejan que la mayoría de empresarios de Norte de Santander no investigan ni desarrollan productos innovadores (Departamento Norte de Santander, 2012). El porcentaje de grupos de investigación del departamento para 2010 respecto del país es de 5.9\% (Departamento Nacional de Planeación, 2010). Estos datos corroboran el desequilibrio de la innovación frente a otras regiones del país.

Un estudio adelantado por la Universidad Francisco de Paula Santander en el sector cerámico destaca la dificultad que tienen las empresas por la falta de capital humano y organizativo que favorezcan los procesos de innovación tecnológica. El aspecto financiero de las empresas es también una debilidad del sector, ya que no existe la suficiente solvencia económica para invertir sostenidamente en actividades de investigación y desarrollo (Ramírez, 2012). 
Otros problemas observados y que afectan la capacidad innovadora de las empresas es la falta de profesionales capacitados; las investigaciones que se adelantan se llevan a cabo de manera informal y no tienen la rigurosidad científica necesaria; hay muy poca articulación con otros actores como el Gobierno, las universidad y los sectores industriales (Ramírez, 2012).

Del análisis de la información presentada, se puede inferir que la situación del departamento es aún más complicada que la del país y que los problemas que impiden el desarrollo de la capacidad innovadora son básicamente los mismos: falta de recursos financieros, falta de personal calificado, trámites excesivos para obtener financiación pública o para acceder a beneficios tributarios y falta de cultura de investigación, ya que los procesos de innovación se realizan de manera informal.

En este sentido y refiriéndose a la situación del país la OCDE (2012) considera que es necesario reconocer que no se dispone hoy de la ciencia y la tecnología que sirvan como herramienta indispensable para el desarrollo social sostenible y que para contar con ellas se debería adoptar políticas adecuadas en corto, mediano y largo plazo.

\section{La normativa}

La normativa colombiana es amplia a la hora de crear y reglamentar instrumentos que permitan abordar con eficiencia el proceso innovador. En la Constitución Política de Colombia, artículo 65 se establece que «el Estado promoverá la investigación y la transferencia de tecnología...» (CPC, 1991), con el propósito de incrementar la productividad. El artículo 71 reza que el "Estado creará incentivos para personas e instituciones que desarrollen y fomenten la ciencia y la tecnología y ofrecerá estímulos especiales a personas e instituciones que ejerzan estas actividades".

La Ley 1286 de 2009 tiene como objetivo general "fortalecer el Sistema Nacional de Ciencia y Tecnología para lograr un modelo productivo sustentado en ciencia, tecnología e innovación, que genere valor agregado a los productos y servicios de la economía y propiciar el desarrollo productivo y una nueva industria nacional".

En el Plan Nacional de Desarrollo 2010-2014 (Ley 1450 de 2011), artículo 3, se proponen como ejes transversales en todas las esferas del quehacer nacional, con el fin de obtener la "Prosperidad para Todos": innovación en las actividades productivas nuevas y existentes, en los procesos sociales de colaboración entre el sector público y el sector privado, y en el diseño y el desarrollo institucional del Estado.

En el "Plan de Desarrollo: Hacia un Estado Comunitario" se plantea la intención de establecer convenios con varios bloques económicos del mundo, para facilitar su cumplimiento de las metas propuestas con el proceso de globalización. El documento CONPES 3297, de 2004 "Agenda Interna para la Productividad y la Competitividad: Metodología" instrumenta los medios para crear las agendas internas regionales a través de un proceso participativo público-privado-académico. 
Mediante el Decreto 0864 del 13 de diciembre de 2007, se creó la Comisión Regional de Competitividad de Norte de Santander, que construye el Plan Regional de Competitividad (PRC) de Norte de Santander, planteando ocho objetivos estratégicos dentro de los que está el de Ciencia y Tecnología al Servicio del Desarrollo. El PRC de Norte de Santander (2007) diseña estrategias que permitan al departamento ser competitivo y sostenible mediante la articulación de acciones entre las instituciones de educación superior, los gremios económicos y el Gobierno.

El Plan de Desarrollo de Norte de Santander 2012-2015 plantea desarrollar modelos productivos en el departamento, apoyados en la investigación que permita crear valor agregado a todos los productos y servicios, para aplicar los resultados de investigación a las soluciones de los problemas de la región. Para ello define estrategias en innovación y tecnología como: crear cultura ciudadana, formular un plan estratégico, promover eventos de divulgación, consolidar el sistema regional de ciencia y tecnología, establecer alianzas público-privadas para desarrollar ciudadelas universitarias, y consolidar políticas de productividad y competitividad en la región.

Como se aprecia los instrumentos de política necesarios para impulsar la innovación en Colombia existen, comenzando por la constitución hasta los planes de desarrollo nacional y locales, no obstante, los indicadores no respaldan esa intención, por ello sería conveniente que en investigaciones posteriores se indague sobre las causas de la ineficacia del sistema y sobre la poca cultura de investigación y desarrollo.

\section{El proceso de medición de la innovación}

Han sido diversos los métodos que se han ideado para medir la innovación, establecer su nivel y realizar trazabilidad de su evolución con el propósito de hacer que las empresas, los sectores y los Estados encuentren las estrategias más adecuadas para lograr competitividad y dar a la sociedad desarrollo y bienestar. Durante los años setenta, la innovación se medía a través de variables como cantidad patentes y gastos en $\mathrm{I}+\mathrm{D}$ de empresas (Albornoz, 2009), pero pronto se entendió que de esa forma no se medía la innovación, sino la oferta de conocimientos o de invención.

Hacia 1976, Keith Pavitt ideó una metodología que permitía medir las actividades de innovación de las empresas, consistente en determinar el porcentaje de recursos destinados a la innovación y la cantidad de productos y procesos que la compañía hubiera introducido (Godín, 2008). En este método no se determina el producto final de la innovación y la generación de nuevos conocimientos, sino se asume que la disposición de recursos económicos es suficiente para crear innovación y desarrollo.

Con la asesoría, orientación y recomendaciones de Pavitt, hacia 1992 la OCDE elabora el Manual de Oslo, el cual permite establecer las fuentes y barreras a la innovación, los cambios en el funcionamiento de las empresas, la naturaleza de las actividades de innovación y los tipos de innovaciones que introducen. Como la innovación se desarrolla en el tiempo, es continuo y permanente, debe medirse sobre 
periodos determinados y tener consideraciones propias de este. En cuanto a los gastos de inversión, se requiere aplicar instrumentos adicionales a los de la contabilidad, ya que las empresas tienen dificultades para reflejarlos expresamente, y con respecto al impacto de la innovación, se debe medir a largo plazo, ya que sus resultados no se ven de inmediato.

El Manual contempla tres tipos de indicadores de innovación: a) de impacto, b) de difusión y c) referente a los costos y gastos. Los indicadores de impacto se miden, teniendo en cuenta la proporción de ventas de nuevos productos introducidos al mercado durante los últimos tres años, por el esfuerzo innovador a partir de la participación en los mercados, por el cambio en las funciones de producción como uso de mano de obra, materias primas, materiales y energía y utilización de capital fijo. Los indicadores de difusión se miden desde la percepción de los usuarios y el relevamiento de uso de tecnologías avanzadas en los procesos manufactureros. Los indicadores referentes a los costos y gastos se miden examinando los gastos incurridos en actividades de innovación en un año dado.

En Colombia, surge el Manual de Bogotá, inspirado en el Manual de Oslo, y desarrollado por Colciencias.

En él se muestra un delicado equilibrio entre el respeto por la sólida e insustituible base conceptual y metodológica que proporcionan los manuales de la OCDE (Oslo y Frascati) y la necesidad de tomar en cuenta las especificidades que caracterizan a los sistemas de innovación de América Latina y el Caribe (Jaramillo, Lugones \& Salazar, 2001. p. 4).

Los indicadores que propone el Manual de Bogotá contienen diez aspectos fundamentales: 1) identificación de la empresa, 2) desempeño económico, 3) actividades de innovación, 4) resultados de innovación, 5) objetivos de innovación perseguidos por la empresa, 6) fuentes de información para la innovación, 7) financiamiento de la innovación, 8) relaciones con el Sistema Nacional de Innovación, 9) factores que afectan la innovación y, 10) evaluación de políticas gubernamentales en materia de innovación, ciencia y tecnología y competitividad.

Con los indicadores propuestos en el Manual de Bogotá, el Departamento Nacional de Planeación (DNP) aplica la primera Encuesta sobre Desarrollo Tecnológico (EDIT) en 1996, con el fin de construir línea de base sobre las características y el comportamiento del desarrollo tecnológico de las empresas colombianas. La segunda encuesta (EDIT II) la realizó el DANE, en el 2005, la tercera (EDIT III), en el 2007 y la cuarta (EDIT IV), en 2013. Las encuetas están desarrolladas de acuerdo con la metodología del Manual de Bogotá.

Desafortunadamente la encuesta no presenta resultados desagregados por departamentos, lo que impide tener una visión aproximada de las realidades regionales, considerando que la caracterización de las economías difiere ostensiblemente entre departamentos. 
A nivel regional, la Universidad Francisco de Paula Santander realizó un estudio sobre la innovación tecnológica en las empresas del sector cerámico del Área Metropolitana de Cúcuta, donde se midieron los siguientes indicadores: 1) capacidad de innovación tecnológica y 2) procesos de innovación (Ramírez, 2012).

Otro método de medición de la tecnología es el propuesto por la Universidad de Zaragoza (España), denominado "Situación y Adecuación Tecnológica (TASI)". Según Torres (2008), el índice TASI2, surge de la experiencia en el ámbito académico e industrial del equipo investigador del Departamento de Ingeniería de Fabricación de la Universidad de Zaragoza, como una adaptación a las condiciones del entorno tecnológico actual. Este método mide doce factores, a saber:

DI: Inversión en investigación, desarrollo e innovación (I+D+I)

AN: Utilización de los sistemas de información, control e internet

IP: Relación con clientes y proveedores

IH: Información de recursos humanos

G: Sistemas de conformación geométrica

SQ: Sistemas de calidad de la empresas

SO: Gestión de la unidad de ingeniería de producto y operativa

AL1. Desarrollo de tecnología y propiedad industrial

AL2. Actividades de innovación

OL1. Gestión del conocimiento

OL2. Gestión de la innovación tecnológica

PX. Destino geográfico de los productos exportados.

El método TASI2 adopta un valor entre 0 y 100 y el criterio para su evaluación es: Tasi $2<25$ nivel tecnológico MALO; $25<=$ Tasi $2<50$ nivel tecnológico DEFICIENTE; $50<=$ Tasi $2<75$ nivel tecnológico ACEPTABLE; Tasi $2=>75$ nivel tecnológico BUENO.

\section{Conclusiones}

Innovación y tecnología son factores fundamentales en la competitividad de la empresa moderna y deben estar en continua adaptación al ambiente globalizado, turbulento y cambiante; su adaptación y desarrollo dependen no solamente de la cultura investigativa e innovadora de la empresa, sino de las condiciones que propicia el Estado a través de políticas públicas, de las relaciones con las universidades, las alianzas estratégicas y de cooperación entre empresas del sector, además del liderazgo de los gerentes que la gobierna. 
La articulación de los factores influyentes en el proceso innovador es responsabilidad del Estado, diseñando políticas que propicien las condiciones para la investigación y canalicen los esfuerzos aislados que realizan los sectores público y privado. Igualmente, las universidades tienen un papel importante, ya que su misión es contribuir con el desarrollo social a través de la investigación y resolución de problemas, y porque aglutinan la mayor cantidad de personas con competencias para la investigación y la innovación.

La innovación se puede dar desde cuatro perspectivas: procesos, productos, mercadeo y gestión. Todos ellos conducentes a generar valor agregado al producto o servicio, que el cliente percibe en términos de calidad, oportunidad o precio, y debe retornar un beneficio a la empresa o sociedad de forma económica, de posición estratégica, prestigio, reconocimiento y bienestar para sus clientes internos y externos. No obstante, la innovación no puede esperar a que se consoliden las modificaciones o los nuevos productos o servicios sino en procura de la búsqueda de conocimientos, inclusive al descubrimiento de nuevas disciplinas científicas, que a mediano y largo plazo, contribuyan al desarrollo económico y social del país. El Estado debe propiciar un sistema efectivo que garantice los esfuerzos de empresa, Estado, universidad y sociedad.

La innovación es un componente fundamental de la competitividad y las empresas deben estar conscientes de ello y tenerla como prioridad a la hora de establecer estrategias de sostenimiento, crecimiento o desarrollo, aprovechando las políticas gubernamentales de ciencia, tecnología e innovación y observando las tendencias mundiales y las posibilidades de aprovechar el éxito innovador en otros países.

Son diversas las fuentes que dan origen a la innovación: generalmente se producen por necesidad del cliente, cambios en el ambiente, como consecuencia de ciclos de vida del producto, por la aparición de tecnologías novedosas, variaciones demográficas, apertura de nuevos mercados, errores en los procesos, información proveniente de otras organizaciones, proveedores, entre otras. Una fuente de innovación muy particular son los centros de investigación de empresas especializadas, las cuales en ocasiones crean necesidades de consumo de productos inexistentes, pero que al salir al mercado producen un nicho importante de negocios.

Sin embargo, no todas las empresas, especialmente las pequeñas, tienen la capacidad y los medios para adelantar procesos innovadores, debido a los costos, a la cultura investigativa o a la disponibilidad de recurso humano calificado, por lo que se debe establecer redes de colaboración y apoyo que permitan interactuar con otras organizaciones, con los gremios y con el Estado para avanzar desde pluralismo y la necesidad común hasta una especificación clara y puntual del proceso en cuestión.

La innovación a nivel global la mide la Organización de las Naciones Unidas; en Colombia lo realiza el Departamento Nacional de Estadísticas, a través de las Encuestas de Desarrollo Tecnológico, aunque no se encuentra desagregada por 
departamentos, por lo que no se conoce los niveles de innovación departamental $\mathrm{y}$, por ende, no se puede realizar seguimiento y trazabilidad al proceso, o medir el impacto de las políticas públicas en materia de innovación y desarrollo.

La última encuesta de innovación tecnológica realizada en Colombia muestra que las empresas mayoritariamente innovan en procesos organizacionales y mejora de productos, un porcentaje muy bajo lo hace en nuevos productos. La mayor fuente de innovación surge a partir de ideas e iniciativas gerenciales, mientras que los departamentos de I+D no aportan significativamente al proceso.

Los principales problemas que presentan investigación es la falta de personal capacitado, falta de recursos e incertidumbre frente a la demanda a las innovaciones. La principal fuente de recursos para la investigación son recursos propios y una mínima proporción de recursos públicos. Para acceder a recursos públicos, las empresas encuentran obstáculos como trámite excesivo, dificultad para cumplir con los requisitos exigidos y desconocimiento de las líneas de financiación pública existentes. Para obtener beneficios tributarios se presentan barreras relacionadas con falta de información, plataforma en línea, dificultad de diligenciamiento, trámites excesivos y poco beneficio en el trámite.

A nivel departamental los indicadores aún son más críticos que el promedio nacional. Las empresas no cuentan las capacidades de capital humano y organizacional que favorezcan la innovación y no hay capacidad financiera para hacer sostenible el proceso. La articulación entre los diferentes actores del sistema es prácticamente nula y eso impide que el sistema obtenga mejores resultados.

\section{Referencias}

Albornoz, M. (2009). Indicadores de innovación: las dificultades de un concepto en evolución. Revista Iberoamericana de Ciencia, Tecnología y Sociedad-CTS 5(13), 9-25.

Argel, G. (2009). Naturaleza y tendencias de la ciencia, tecnología e innovación (CTI) en América Latina y el Caribe. Un análisis crítico al modelo institucional colombiano. Pensamiento \& Gestión 27, 1-323.

Arrow, K. (1962). The economic implications of learning by doing. Review of Economic Studies 22(1), 1-80.

Arzola, M. (2007). Estrategias tecnológicas para la industria transformadora de productos de bienes de consumo de aluminio en Venezuela. Universidad, Ciencia y Tecnología 11(42), 1-20.

Benavides, C. (1998). Tecnología, innovación y empresa. Madrid: Pirámide.

Boza, M., \& Pérez, R. (1996). Seguridad jurídica y competitividad. Caracas: IESA. 
Castells, M. (1999). La era de la información. En Economía, sociedad y cultura, la sociedad red. México: Siglo Veintiuno.

Comisión Regional de Competitividad Norte de Santander. (2007). El Plan Regional de Competitividad de Norte de Santander. Cúcuta: Publi Impresos JAR.

Damanpour, F., \& Gopalakrishnan, S. (1998): Theories of organizational structure and innovation adoption: The role of environmental change. Journal of Engineering and Technology Management 15, 1-24.

Departamento Administrativo de Ciencia y Tecnología - Colciencias. (2010). Indicadores departamentales de ciencia, tecnología e innovación 2010. Norte de Santander. Recuperado de http://www.ocyt.org.co/html/ boletines/ NorteSantander.pdf.

Departamento Administrativo Nacional de Estadística - DANE. (2011). Encuesta de Desarrollo e Innovación Tecnológica, Servicios y Comercio. EDIT III. Bogotá: DANE.

Departamento Administrativo Nacional de Estadística - DANE. (2013). Encuesta de Desarrollo e Innovación Tecnológica, Servicios y Comercio. EDIT IV. Bogotá: DANE

Departamento Nacional de Planeacion - DNP. (2010). Indicadores Departamentales de ciencia, tecnología e innovación 2010. Bogotá.

Departamento Norte de Santander. (2012). Plan de Desarrollo para Norte de Santander 2012-2015 (2012). Un Norte Pa'lante (pp. 1-179). Gobernación Norte de Santander. Cúcuta. Colombia.

Drucker, P. (2002). The discipline of innovation. Harvard Business Review (pp. 1-11). Reference R0208F.

Dussauge, P., Hart, S., \& Ramantsoa, B. (1992). Strategíc technology management. Chichester, England.

Edquist, C., \& Johnson, B. (1997). Institutions and organizations in systems of innovation. In Edquist, C. (Ed.). Systems of innovation: technologies, organizations, and institutions. London: Pinter Publishers/Casell Academic.

Enright, M., Frances, A., \& Scott, E. (1994). Venezuela, el reto de la competitividad. Caracas: IESA.

Forrest, E. (1991): Models of the process of technological innovation. Technology Analysis \& Strategic Management 3(4), 439-453.

Freeman, C. (1991). Networks of innovators: a synthesis of research issues. Research Policy 1991, 499-514. 
Gallouj, F., \& Weinstein, O. (1997). Innovation in services. Research Policy, 26, 4-5; 537-556. Recuperado de http://dx.doi.org/10.1016/S0048-7333(97)00030-9.

Guevara, L. (2000). Incidencia de la tecnología blanda y la tecnología dura en el desarrollo industrial de la biotecnología en Colombia. Innovar, Revista Ciencias Administrativas y Sociales, 79-93. Bogotá, Colombia.

Haguenauer, L. (1989). Competitividade, conceitos e medidas. uma resenha da bibliografia recente com êmfase no caso brasileiro. Revista de Economía Contemporánea, 1-30. Río de Janeiro. Brasil.

Hobday, M. (2005). Firm-level innovation models: Perspectives on research in developed and developing countries. Technology Analysis \& Strategic Management, 17(2), 121-146.

Jaramillo, H., Lugones, G., \& Salazar, M. (2001). Normalización de indicadores de innovación tecnológica en América Latina y el Caribe. Bogotá: RICYT/ OEA/ Colciencias/OCYT.

Kline, S., \& Rosenberg, N. (1986). An overview of innovation. In The positive sum strategy: Harnessing technology for economic growth. Landau, R., y Rosenberg, N. (Ed.). Washington, D.C: National Academy Press.

Leif, C. (1999). Systems of innovation: theory and policy for the demand side. Technology in Society 21, 63-79. Department of Technology and Social Change, University of Linköping, S-581 83 Linköping, Sweden.

Listerri, J., y Angelelli, P. (2002). Guía operativa para programas de competitividad para la pequeña y mediana empresa. Informe de trabajo. Washington: Banco Interamericano de Desarrollo.

López, N., Montes, J., Vázquez, C., y Prieto, J. (2004). Innovación y competitividad: implicaciones para la gestión de la innovación. Revista Tribuna de Debate 24, $1-25$.

Ministerio de Industria y Turismo de Colombia. (2014). Información suministrada por el autor al Ministerio mediante radicado solicitud $\mathrm{N}^{\circ}$ 1-2014-007792. Recuperado de https:/gestiondocumental.mincit.gov.co/ gestiondoc/modulodocumental/documentos/Radicado/Documento\%20 Anexo\%20Expediente/Respuesta\%20a\%20Se\%C3\%B1or\%20Julio\%20 Alfonso\%2014-005-2014\%20(16-07-2014)7182014123855\%20PM.pdf

Nagles, N. (2007). La gestión del conocimiento como fuente de innovación. Revista Escuela de Administración de Negocios, 61, 77-87.

Ochoa, M. (2007). Innovación, tecnología y gestión tecnológica. ACIMED. Recuperado de http://eprints.rclis.org/10618/1/08-Innovaci\%C3\%B3n,tecnolog\% C3\%ADaygesti\%C3\%B3ntecnol\%C3 \%B3gica.pdf. 
Organización de Estados Iberoamericanos para la Educación, la Ciencia y la Cultura (OEI). (2012). Ciencia, tecnología e innovación para el desarrollo y la cohesión social. Madrid: OEI

Organización para la Cooperación y el Desarrollo Económico. OCDE (2005). Manual de Oslo. Guía para la recogida e interpretación de datos sobre innovación (p. 1-194). OECD/European. Communities.

Peñaloza, M. (2007). Tecnología e innovación, factores claves para la competitividad. Actualidad Contable Faces 10(15), 82-94.

Pineda, L. (2009). Enfoques alrededor de la gestión estratégica de la innovación. Facultad de Administración, Universidad del Rosario. Bogotá.

Porter, M. (1990). The Competitive Advantage of Nations. The Free Press. ISBN $0684841479,9780684841472$.

Ramírez, P. (2012). La innovación tecnológica en las empresas del sector cerámico del Área Metropolitana de Cúcuta. Revista Respuestas 17(1), 66-77.

República de Colombia. (1991). Constitución Política de 1991. Bogotá: Diario Oficial.

República de Colombia. (2009). Ley 1286 de 2009, Por medio del cual se fortalece el Sistema Nacional de Ciencia y Tecnología. Bogotá: Diario Oficial.

República de Colombia. (2011). Ley 1450 De 2011, Por la cual se expide el Plan Nacional de Desarrollo, 2010-2014. Bogotá: Diario Oficial.

Ricyt. (2012). Indicadores Iberoamericanos de Ciencia y Tecnología, Red de Indicadores de Ciencia y Tecnología - RICyT. www.ricyt.org. Consultado febrero de 2013.

Rothwell, R. (1994): Towards the fifth-generation innovation process. International Marketing Review 11(1), 7-31.

Schumpeter, J. (1978). Teoría del desenvolvimiento económico. México: Fondo de Cultura Económica.

The Business Scholl for the World, World Intellectual Property Organization. (2012). The Global Innovation Index 2012. Stronger Innovation Linkages for Global Growth. Soumitra Dutta, INSEAD. Editor. Francia.

Torres, F. (2008). Método para estimar el nivel tecnológico de las empresas: índice de situación y adecuación tecnológica, TASI. Laboreo, Santiago Departamento de Ingeniería de Diseño y Fabricación. Universidad de Zaragoza. España.

Von Hippel, E. (1988). The sources of innovation. Oxford: Oxford University Press. 